\title{
Radio over Fiber Technology
}

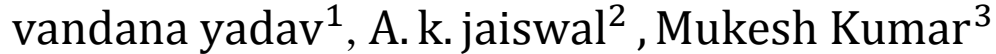 \\ (M.tech. Scholer,dep. Of Ece/Shiats, India),(pro. \& h.o.d. dep. of Ece/Shiats,India),(Assistant pro. Dep. Of \\ Ece/Shiats,India)
}

\begin{abstract}
Radio over fiber is an analog transmission in which radio frequency is modulated with laser diode. It is an integration of wireless and fiber optic network.Today the peek requirement is capacity of the channel so optical communication has come in picture. The future provision of multimedia and broadband the radio over fiber systems are good option. In this paper, four signals are transmitted for $120 \mathrm{~km}$. through single mode fiber with erbium doped fiber amplifier. Two base stations are introduced at $40 \mathrm{~km}$. each to add and drop a particular signal. The well known advantages of fiber such as low loss, light weight, large bandwidth, small size, low cable cost are very useful in optical communication. Warless sensor network is used where battery recharge or replacement is impossible so network life time is more important. In this paper we are presenting a heterogeneous network in which various energy efficient technologies are used.It is an essential technology for the provision of access to broadband wireless communication in a range of application. Their transmission properties are very useful in their implementation as the backbone of a wireless network.
\end{abstract}

Keyword: Erbium-doped fiber, central station, Optic-system software, Radio over fiber, semiconductor,

\section{Introduction}

Optical communication is a form of communication that uses light as the transmission medium. Radio over fiber [1] is an analog optical link transmitting modulated RF signals. It serves to transmit the RF signal to and from central station to base station. The main requirements of radio over fiber link architecture are duplex operation (i.e., downlink-uplink) [1] reasonable length, need a few millimeter-wave components only in the base stations and also need of only few high performance optical components.

RoF systems are now being used for enhanced cellular coverage inside buildings. It has introduced as a cost effective approach for reducing radio system costs because it simplifies the remote antenna sites and enhances the sharing of expensive radio equipment located at central stations. The frequencies of the radio signals distributed by RoF systems span a wide range (usually in the $\mathrm{GHz}$ region) and depend on the nature of the applications.

\section{Radio Over Syestem}

Radio over Fiber (RoF) is an optical fiber link to distribute modulated RF signals from a central location to remote antenna units. The RoF systems are introduced to replace a central antenna with a low power distributed antennas system (DAS). RoF system is consisting of many base stations, which are connected to a single central station. RoF systems centralize the RF signal processing function in one shared location, and use optical fiber link to distribute the RF signals to the RAUs or BSs [2]. RoF based wireless access network architecture is proposed, as a promising alternative to broadband wireless access network. In network architecture, the CS performs all switching, routing and network operations maintenance. Optical fiber network interconnects a number of simple and compact antenna BSs for wireless distribution.

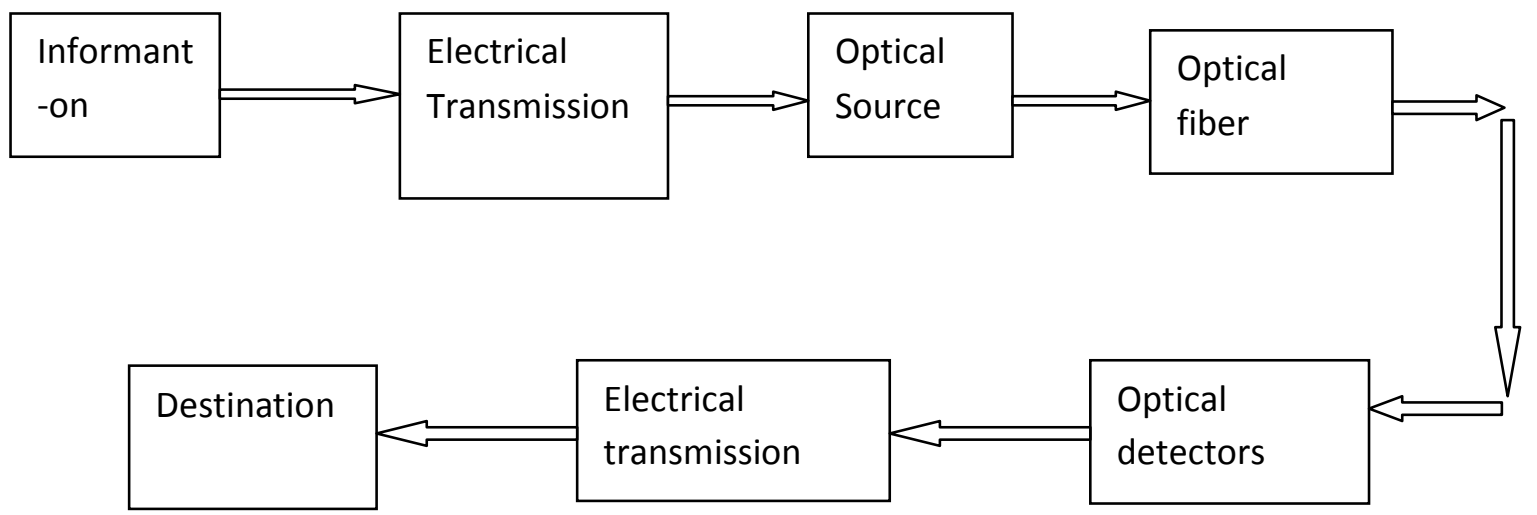




\section{Application of radio on fiber}

- Cellular network: mobile traffic is relayed between base station and central station via ROF system.[3]

- Wireless LANs: can be used to distribute wireless LAN signals operating at $2.4 \mathrm{GHz}$ to $5 \mathrm{GHz}$.[3]

- Video distribution system: it can be used for MANs, wired and wireless network.[2]

- Vehicle communication and control: ROF can be used for intelligent transport system, road- tovehicle communication system.[2]

\section{Advantages of Radio over Fiber system}

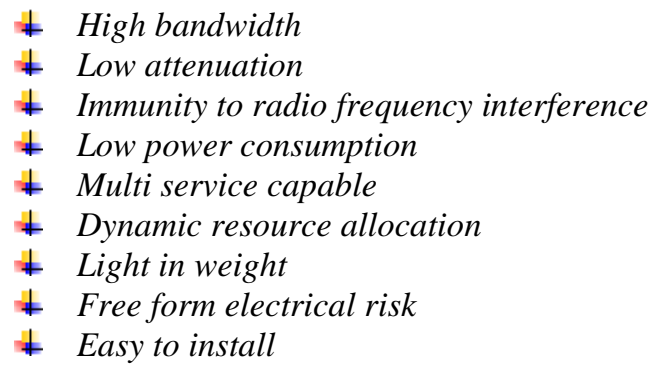

\section{Introduction of different components used in ROF system}

\section{Wavelength division multiplexing:}

WDM [4] is used to multiplex the different optical signals on a single optical fiber by using different wavelengths of laser diode. . This allows for a multiplication in capacity of an optical fiber by adding new channels, each channel on a new wavelength of light, in addition to enabling bidirectional communications over one strand of fiber. This is a form of frequency division multiplexing (FDM) but commonly it is called wavelength division multiplexing.

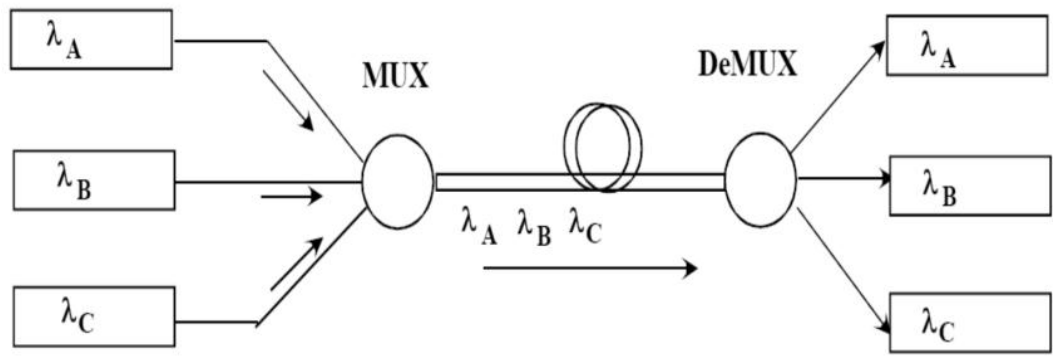

Fig 5.1 wavelenght division multiplexing

optical add-drop multiplexer

OADM [5] is a technique that is used in WDM for multiplexing and routing different optical signals to or from the optical network. It consist of optical multiplexer or optical demultiplexer, a path between optical mux and optical demux, a set of ports for adding and dropping optical signals , a specific wavelength is added or dropped by circulator and FBG (fiber brag grating)s

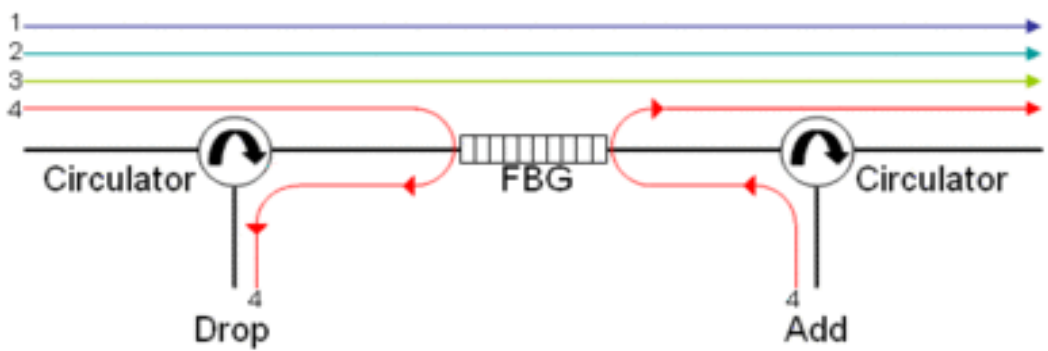

Fig.5.2 optical add- drop multiplexing

\section{Erbium doped fiber amplifier}

EDFA [6] is a optical amplifier used in optical communication. When optical signals are transmitted over a hundreds of kilometer then it require amplification. In optical communication there may be in-line, pre and post amplification. EDFAs are used for long distance communication with fiber loss less than $0.2 \mathrm{db} / \mathrm{km}$. For wavelength near to $1550 \mathrm{~nm}$ 


\section{Laser diode}

A laser diode is a device whose active medium is a semiconductor similar to that found in a LED. The most common type of laser diode is formed from a Ntype and Ptype material as p-n junction which is powered by injected electric current .A laser diode is formed by doping a very thin layer on the surface of a crystal wafer. Laser diodes form a subset of the larger classification of semiconductor p-n junction diodes. Due to forward biasing two type of charges are introduced in device. Holes are injected from the p-doped, and electrons from the n-doped, semiconductor.

\section{Photodetecter}

When a photon with energy greater than the band gap Eg is incident on the semiconductor, this energy is absorbed by the material and generates an electron-hole pair that is an electron in the conduction band and a hole in the valence band. When the pair is created within the space charge region, the electric field in the junction separates the charges and drifts them to the neutral regions. The carrier drift generates a photocurrent in the external circuit that provides an electrical signal. The photocurrent lasts the time needed for the electron and hole to cross the depletion layer and reaches the neutral regions. When the drifting hole reaches the p-type region it recombines with an electron entering the $\mathrm{p}$ side form the negative electrode that is from the power supply. .

\section{Simulation setup and design analysis}

In this proposed method two data signals are generated by two laser diode having different frequencies which feeds to Mach-Zender modulator [7] where incoming signal from pseudo-random bit sequence mixed with NRZ signal is modulated with it. And two- tone video signals are generated by combing of two analog signals with different frequency that is $500 \mathrm{MHZ}$ mixed with $525 \mathrm{MHZ}$ by directly modulated laser diode and second video signal is combined with $600 \mathrm{MHZ}$ with $625 \mathrm{MHZ}$. These four signals are multiplexed by WDM and amplified by EDFA and then these multiplexed signals are transmitted to single mode fiber for $40 \mathrm{~km}$. At base station one required signal $(1552 \mathrm{~nm}$.) is dropped and then $1552 \mathrm{~nm}$. Is added by OADM after each 40 $\mathrm{km}$.

At the receiver side all these signals are demultiplexd with DWDM, now these four signals feed on four different photodetecters and shown on different visualizer.

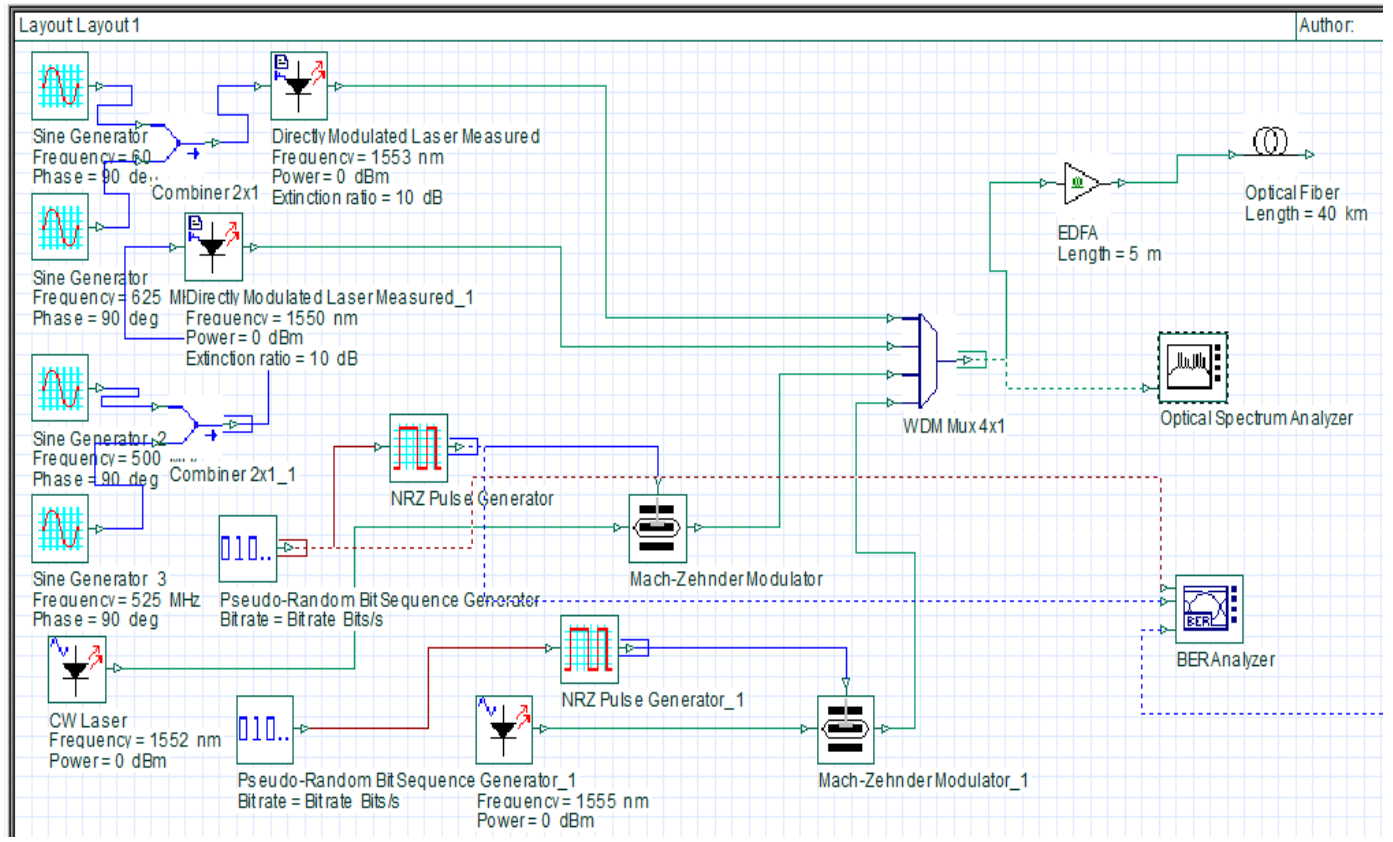

Fig.6.1 Central station 


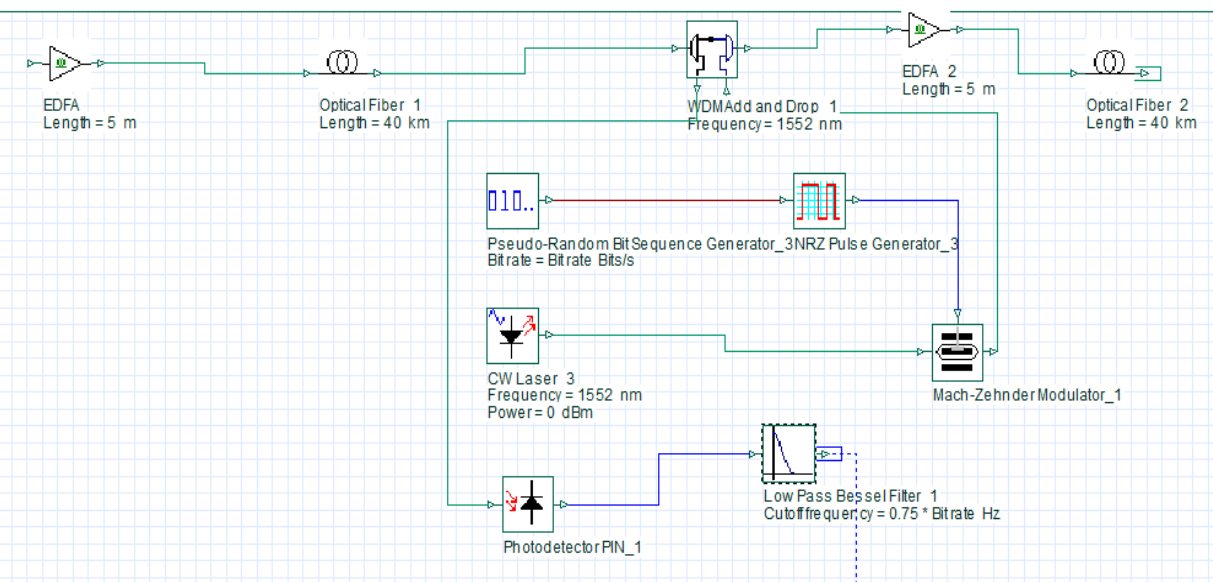

Fig 6.2 base station

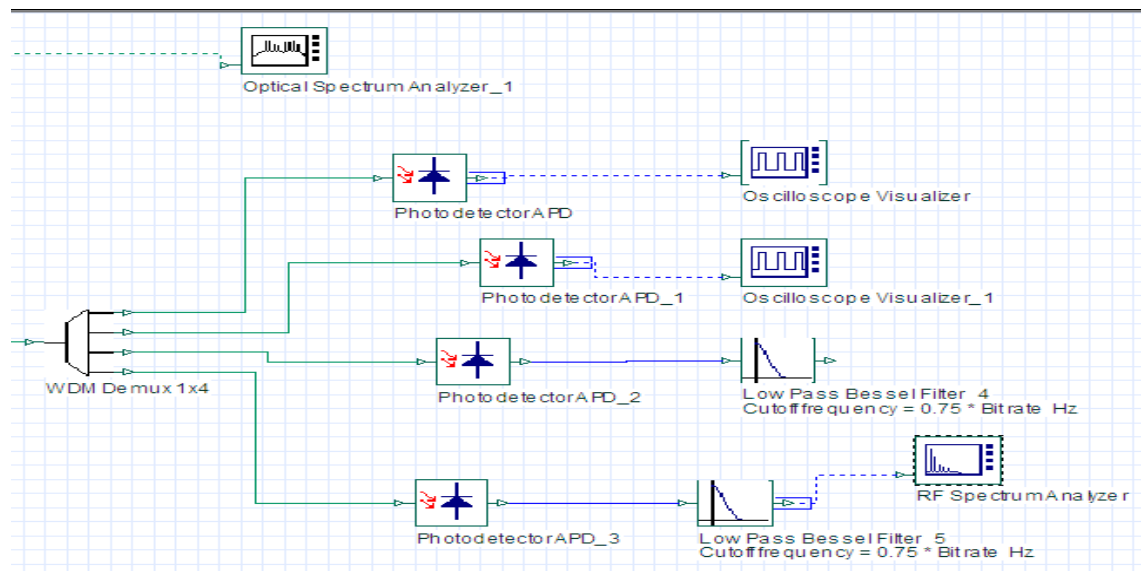

Fig 6.3 Receiver Station

\section{Result and discussion}

Four signals $1550 \mathrm{~nm}, 1552 \mathrm{~nm}, 1553 \mathrm{~nm}, 1555 \mathrm{~nm}$ is shown in optical spectrum analyzer at the output of MUX in the central station as in fig.7.2. And video signal is shown in fig.7.1. The whole Radio over Fiber system consists of wavelength division multiplexing and optical add-drop multiplexing techniques. This is simulated using Opt system 10. WDM technique is used to transmit four different signals through a single fiber for long distance. OADM technique enabled us to transmit both down-link and uplink data via the same singlemode fiber. EDFA is used to amplify the signals after every $40 \mathrm{~km}$ of the transmission link. . At the receiver station some noise signals are presented with required signals but desired signals are detected easily due to higher amplitude of that signals. The proposed system is suitable for high bit rate and long-haul microwave optical links.

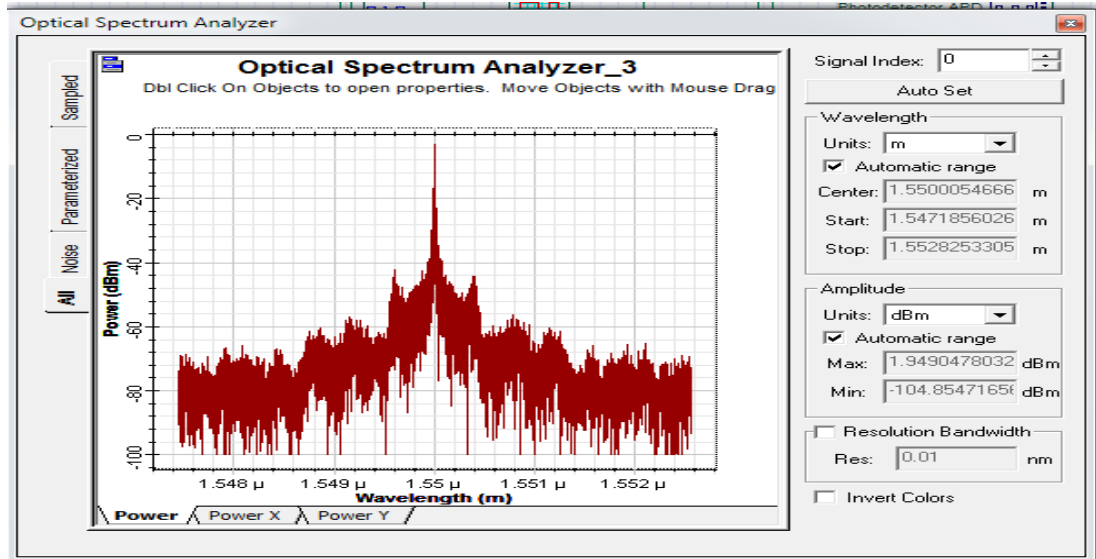

Fig 7.1 Video signal at the central station 


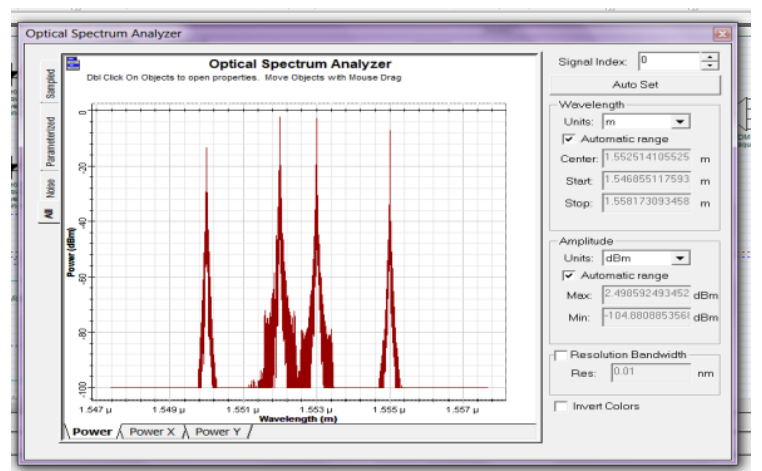

Fig 7.2 Transmitted signals at the central station

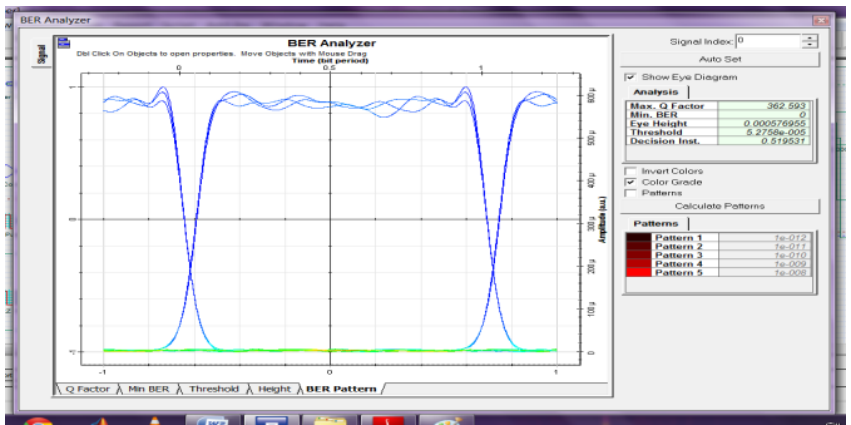

Fig 7.3 Eye diagram at base station

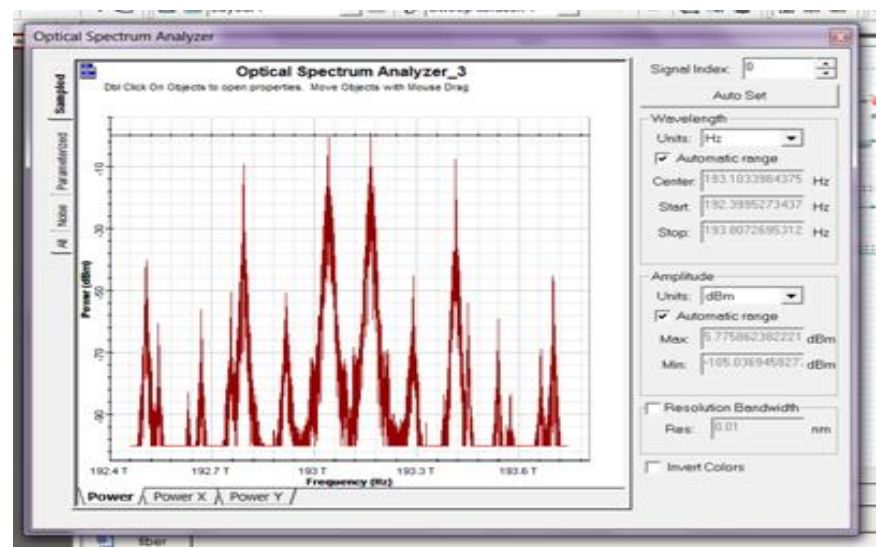

7.4 Received signals at the receiver

VIII. Conclusion

In this paper we have visualized four signals at MUX fig.7.2, video signal at the Central station fig.7.1 and eye diagram at base station fig.7.3 then these signals is presented at the receiver which is shown in optical spectrum analyzer as in figure 7.4.This proposed technique full duplex data and two-tone video signal consist of wavelength multiplexing and optical add -drop multiplexing is stimulated on optisystem 10 .WDM is used for multiplexing of different four signals to transmit on single fiber.s

\section{References}

[1] Smith G.H. et al [1998], "A Millimeter-Wave Full-Duplex Fiber-Radio Star-Tree Architecture Incorporating WDM and SCM" IEEE Photonics Technology Letters, Vol. 10, No.11

[2] Lu Hai-Han et al [2007] “A Full-Duplex Radio-on-Photonic Crystal Fiber Transport System,” IEEE Photonics Technology Letters, vol. 19, No. 11.

[3] Lu Hai-Han et al [2008] "Full-Duplex Radio-on-Fiber Transport System Based on Main and Multiple Side Modes Injection-Locked DFB Laser Diode" Institute of Electro-Optical Engineering, National Taipei University of Technology.

[4 ] Pham Thang T. et al [2008]" Bidirectional 1.25-Gbps WDM-PON with Broadcasting Function Using A Fabry-Perot Laser Diode and RSOA “, Journal of the Optical Society of Korea Vol. 12, No. 4, December 2008, pp.

[5] 11Ji Ho-Chul et al [2009] "Full-Duplex Radio-Over- Fiber System using Phase-Modulated Downlink and Intensity- Modulated Uplink". IEEE Photonics Technology Letters, Vol. 21, No. 1

[6] Gabla P. M. et al [2009] "1111 km, Two-Channel IM-DD Transmission Experiment at 2.5 Gb/s Through 21 In-Line Erbium-Doped Fiber Amplifiers", IEEE Photonics Technology Letters, Vol. 21, No. 1

[7] Pham Thang Tien et al [2009], "Bidirectional 1.25-Gbps Wired/Wireless Optical Transmission Based on Single Sideband Carriers in FabryPerot Laser Diode by Multimode Injection Locking”, Journal of Light wave technology, vol. 27, No. 13 